\title{
Cognitive Deficits in Primary Brain Tumours: A Framework for Management and Rehabilitation
}

\author{
Michael Back ${ }^{1,2}$, Elizabeth Back ${ }^{3}$, Marina Kastelan ${ }^{1}$, Helen Wheeler ${ }^{1,2}$ \\ ${ }^{1}$ Northern Sydney Cancer Centre, Royal North Shore Hospital, Sydney, Australia; ${ }^{2}$ Northern Clinical School, Sydney Medical School, \\ University of Sydney, Sydney, Australia; ${ }^{3}$ Occupational Therapy Clinic, North Shore Medical Centre, Sydney, Australia. \\ Email: michael.back@health.nsw.gov.au
}

Received November $11^{\text {th }}, 2013$; revised December $10^{\text {th }}, 2013$; accepted December $18^{\text {th }}, 2013$

Copyright (c) 2014 Michael Back et al. This is an open access article distributed under the Creative Commons Attribution License, which permits unrestricted use, distribution, and reproduction in any medium, provided the original work is properly cited. In accordance of the Creative Commons Attribution License all Copyrights (C) 2014 are reserved for SCIRP and the owner of the intellectual property Michael Back et al. All Copyright (C) 2014 are guarded by law and by SCIRP as a guardian.

\section{ABSTRACT}

Cognitive deficits related to tumour or treatment are estimated to be present in the majority of patients diagnosed with a primary brain tumour, with a large variation based on tumour site, extent, and grade, as well as nature of intervention. These deficits have a significant impact on patient's quality of life and functional status. The improved patient survival duration in recent years has increased the need to detect, understand and intervene on these cognitive deficits, and optimise patient pathways for treatment. This article reviews the nature of deficits arising in patients with primary brain tumour, and the association of the deficits with neuroanatomical site of tumour. The approach to interventions through adaptation and remediation is outlined, with aim of improving function, promoting generalization of outcomes and optimizing any neuroplasticity that may arise. The evidence for pharmacological therapies and cognitive rehabilitation is presented, and a framework for implementation is provided.

\section{KEYWORDS}

\section{Glioma; Cognitive; Rehabilitation}

\section{Introduction}

Recent advancements in the multidisciplinary management of brain tumours have resulted in significant improvements in survival duration [1]. There is a vast natural history for tumours including prolonged survival beyond 10 - 15 years expected for the low to intermediate grade tumours [2,3]. Even for the patient subgroup diagnosed with glioblastoma multiforme the median survival has increased from 9 - 12 months in the years before the introduction of temozolomide in 2006, to 18 - 24 months in patients currently being managed [1]. This has meant patients not only have longer survival to manage with the side effects of tumour at initial diagnosis and its subsequent treatment, but also increased duration to develop late side effects of intensified treatment multidisciplinary modalities.

Whilst established rehabilitation services have incorporated physical therapies for patients with brain tumours, the options for neurocognitive deficits remain poorly studied, resourced and utilized [4]. This review aims to provide a structure to approach neurocognitive deficits in patients with primary brain tumours with a framework to interventions.

\section{Incidence and Impact of Cognitive Deficits}

Cognitive impairment in patients with brain tumours is likely to be influenced by tumour site, laterality, grade and nature of interventions. Diagnosis may only be evident on neuropsychological testing or close interviews by patient and carers. Most impairment will be left unexplored or untreated $[4,5]$. The limited historical data suggests that specific impairments are present in at least $30 \%$ of patients' post-surgical procedure and extending up to $90 \%$ at long term follow up post treatment [6-8]. A recent prospective study by Johnson assessed 80 patients with glioblastoma in the immediate postsurgical period 
and confirmed deficits in memory in $60 \%$ and executive function in 53\% of patients [7].

Different to the deficits occurring after other brain pathology such as in traumatic brain injury, the impact of anti-cancer therapies may induce progressive deterioration over time after therapy. Late follow-up in patients with low-grade glioma has demonstrated worsening of neurocognitive status at year's 5 - 10 post therapy that was not evident at earlier review [9].

The cognitive deficits have been demonstrated to be a major factor in health related quality of life endpoints especially when resulting in loss of functional independence. The resulting in impact on ability to undertake activities of daily living, reduced autonomy and inability to return to work may create an even greater restriction than a physical disability [10].

The natural history varies significantly between tumour grade and sites. Rapidly progressive tumours such as glioblastoma may present with acute symptoms related to raised intracranial pressure or vascular event. The volume of tumour may be small if sited in an eloquent area compared to a non-eloquent site given the early presentation of symptoms. Slow growing tumours such as lowgrade glioma or anaplastic oligodendroglial tumours may have more extensive brain infiltration but subtle or minimal acute symptoms perhaps masked as part of underlying personality.

The aetiology of cognitive deficits following a diagnosis of brain tumour may also be multifactorial with other potential reported exacerbating factors being treatment related lethargy, endocrine dysfunction, anticonvulsant therapy, and mood disorders [6]. Anticonvulsant medication has been associated with significant impairment of working memory and executive function in a study of 156 relapse free long-term survivors of low-grade glioma [11]. A multidisciplinary approach to assessment of cognitive deficit is important to exclude other meeting causes of impaired function.

\section{Nature of Neurocognitive Dysfunction}

\subsection{Classification}

Extrapolated from Traumatic Brain Injury, cognitive deficits may be appraised in the nature of the deficit or the neuroanatomical site in the brain that is affected. Deficits at time of diagnosis may be limited to the region of brain affected, though after treatments such as radiation therapy there may be more global deficits with extension beyond the neuroanatomical boundary. Neurodegeneration along nerve tracts and diachesis, where a secondary area of the brain loses function because of injury to its dependent region, may also contribute to extension beyond predicted regions.

Correspondingly a classification of cognitive impair- ment into deficit based or neuroanatomical region based can aid in the assessment or prediction of impairment.

\subsection{Deficit Based Classification}

\subsubsection{Executive Function}

This relates to the coordination of learnt skills that are required to achieve a goal or process, especially in new or unstructured situations. These skills include initiation, planning, organization, sequencing, problem solving and decision-making. There is a knowledge base that is required, and then a system to execute the process. It is intimately linked with working memory to mobilize the knowledge and metacognition that self-monitors the process. It is critical to task planning and completion in activities of daily living. Impairment of frontal lobe, especially in the dorsolateral prefrontal cortex, is associated with disturbance of executive function.

\subsubsection{Attention Deficits}

This relates to the ability to select and process sensory information that are relevant for a process. It may be focused (respond to different kinds of sensory input); sustained (vigilant and maintain without distraction); selective (discriminate between different stimuli); alternating (move between tasks); and divided (ability to undertake several tasks) [12]. Attention deficits may arise in frontotemporal lobe damage as well as limbic system; and secondary effects will arise in memory and future executive functions if attention is impaired.

\subsubsection{Memory}

This is the process by which a perception or information is encoded, stored and retrieved. Memory can be subcategorised into different processes: Working (short term storage and manipulation of information to be activated for immediate task completion); Implicit or Explicit (subconscious or, learned respectively); Declarative or Procedural (conscious recollection or subconscious acquisition of skills respectively); Prospective (remember future tasks); Semantic (factual knowledge); Episodic (recall of previous experience) and Long-Term (permanent storing of information) [12-14]. The anatomical sites that relate to memory encoding are diffuse in the brain but specifically the medial temporal lobe and frontal cortex appear to be key sites for the processing, retention and then retrieval of memory.

\subsubsection{Metacognition}

This relates to a person's understanding and monitoring of their own cognitive processes. It is an important component of attention and executive function, involved with generalization, self-regulation and self-awareness. The prefrontal cortex, specifically the inferior or lateral orbitofrontal may be associated with this loss of self-moni- 
toring [13].

\subsubsection{Body Scheme Disorders or Neglect}

This is the loss of the perception of body position and relationship. It predominantly impacts on left side of body from injury in the right parietal lobe, and is most notably demonstrated in unilateral body neglect. It can also manifest as autotopagnosia (lack of awareness of body parts), right-left discrimination (inability to distinguish laterality) and finger agnosia (bilateral poor finger dexterity).

\subsubsection{Agnosia}

This refers to lack of recognition of familiar objects that occurs due to disturbance in sensory processing. Agnosia may be visual (visual stimuli), tactile (texture and form), colour (distinguishing colour) and auditory (sounds). It may also be more specific with prosopagnosia (known faces), topographagnosia (maps and plans) and environmental (direction in familiar places). The site of damage may be related to both hemispheres, as object recognition requires both visual and perception processing.

\subsubsection{Apraxia}

This relates to motor programming disorders and is the inability to perform purposeful movements. There is an impact on planning and execution of a function. It may be ideomotor (imitation or commands); ideational (execution of idea or loss of memory for movement); or constructional (producing designs). The aphasias can also be included in this group as losses of speech planning (receptive aphasia) or speech production (expressive aphasia) are part of a motor programming disorder. The apraxia arises from injury to the left parietal lobe, though motor planning can arise in either hemisphere.

\subsubsection{Visuospatial Disorder}

This is a loss of the perception or rapid processing of visual stimuli from the environment. It can occur due to any impact along the visual pathway from lens to optic radiation in the occipital lobe. Visual field deficits are to be distinguished from visual inattention with the latter being a body scheme disorder and more frequent in right hemispheric injury.

\subsection{Neuroanatomical Site Classification}

Cognitive deficits related to brain tumours differ from other pathologies such as TBI or ischemia as the onset may be gradual rather than acute and the injury quite focal and regionally defined. However the presence of oedema or diachesis may result in a more diffuse area being affected, as would morbidity related to radiation therapy or chemotherapy. Neuroanatomical regions affected may be considered in regards to laterality, as well as cortical and deep regions.

\subsection{Laterality}

Right hemispheric or non-dominant lobe lesions may have deficits relating to neglect or impairment in attention and insight.

Left hemispheric or dominant lobe lesions are more likely to have apraxia, executive function loss of sequencing and organisation, expressive and receptive aphasia.

\subsection{Cortical Sites}

\subsubsection{Frontal Lobe}

The frontal lobe can be divided into the prefrontal cortex, and posterior premotor, supplementary motor and primary motor cortex. For cognitive deficits the prefrontal cortex, especially on the right hemisphere, is the key regulator for executive functions, metacognition and attention. The proximity to motor cortex can provide the organisation and regulation of actions by integrating cognitive and sensorimotor information [13]. The prefrontal cortex can be divided into three regions.

1) Orbitofrontal: injury to this region may result in disinhibition, poor impulse control, impaired attention and emotional lability.

2) Dorsolateral: Decision-making, organisation and sequencing may be affected. Self-monitoring and awareness as part of metacognition may be impaired. It also is an important region for integrating working memory.

3) Medial Frontal: Motivation and attention impairment, with subsequent apathy and slowed response.

\subsubsection{Temporal Lobe}

The medial temporal lobe structures are crucial for memory processing and retrieval. Injury to the hippocampus impacts on declarative or conscious memory recall, as well as new memory formation. Procedural learning may be maintained given that activity is based in subcortical structures, but working memory can be impacted given the limited ability to form new networks. Emotional regulation may be affected with disturbance of other medial structures such as amygdala and uncus. Superior based lesions in the dominant lobe may affect receptive language with involvement of Wernicke's area.

\subsubsection{Parietal Lobe}

Disruption to motor and sensory pathways may occur with injury to parietal lobe. Left hemispheric lesions may give rise to apraxia, and right-sided lesions may cause visuospatial impairment.

\subsubsection{Occipital Lobe}

Lesions may predominantly cause visual field loss, and impaired visual recognition. 


\subsection{Non-Cortical Sites}

\subsubsection{Anterior Thalamus}

Injury to these regions may interrupt the neuronal information from cortex to brainstem and thus cause contralateral hemisensory and motor disturbance, apraxia or neglect. If the internal capsule is affected then motor pathways are affected. Significant injury may also affect alertness with hyper somnolence and behavioral disturbance. Loss of procedural memory and working memory is also impacted.

\subsubsection{Posterior Thalamus}

This site may impact on sensory regulation especially in facial or upper limb sites; as well as aphasia and neglect. Altered consciousness can also occur.

\subsubsection{Caudate}

Confusion, agitation or apathy may arise with injury to the deep nuclei. Speech disturbance with dysarthria and working memory disturbances may also be present.

\subsubsection{Putamen}

Contralateral motor and sensory disturbances can arise, as well as disturbed consciousness and conjugate gaze palsies.

\subsubsection{Pons}

Brainstem effects of bilateral motor and sensory changes, gaze palsies, and consciousness may arise.

\subsubsection{Cerebellum}

Lesions may impact on coordination and limb ataxia.

\section{Approaches to Rehabilitation}

Specific interventions for Cognitive Rehabilitation traditionally have been classified into an approach that is either adaptation (compensatory) or remediation (restorative) [12]. Adaptive therapies are based on an understanding of the symptoms resulting from the deficit and utilize strategies to improve function. It does not aim to alter the underlying impairment but can minimize the impact of the deficit. Remedial interventions are aimed at reducing the severity of the deficit and focuses on the cause rather than the effect of a deficit. Through activities such as process training it aims to promote neuroplasticity or allow neuronal structures to reorganize and subsequently improve function.

Table 1 summarises a structure to the programme as detailed by the Society for Cognitive Rehabilitation [15]. A multidisciplinary rehabilitation programme is likely to involve both adaptive and remedial strategies.

Additionally as described by Sohlberg and Mateer (2001) there should be an emphasis to provide functional endpoints to a rehabilitation programme, so that impact on activities of daily living can be optimized. The ultimate measure of success of any cognitive rehabilitation program is improvement in an individual's ability to manage work, daily living or leisure activities, not simply on practiced therapy tasks [16]. This will involve individualising both adaptive and remedial interventions to processes that are relevant to the patient's home and work environment.

Educational and metacognitive strategies are also important as part of an overall cognitive rehabilitation approach as patient understanding, insight and awareness of deficits will be necessary to consolidate any intervention and allow application to their own lifestyle and environment. Utilising feedback, goal setting and self-monitoring are metacognitive approaches that aid in engagement and motivation [17].

\section{Interventions}

\subsection{Pharmacological}

Medical therapy has been used to compensate for functional loss and perhaps to provide some restoration of the deficit. The available data is limited and phase III studies have not confirmed any significant global benefit in functioning; though certain cognitive domains on testing have improved such as executive function [6]. Interventions should only be considered for symptomatic patients, especially if lethargy is associated with the functional deficits. There is no data to demonstrate a protective or remedial effect for patients with no deficit.

Table 1. Framework of interventions for cognitive impairment as adapted from society of cognitive rehabilitation [15].

\begin{tabular}{|c|c|c|}
\hline Interventions & Aim & Principles \\
\hline Educational & $\begin{array}{l}\text { Develop understanding and awareness into the } \\
\text { nature of the deficit. }\end{array}$ & $\begin{array}{l}\text { Improve the insight into the effect of the deficit, as well as metacognitive } \\
\text { processes to aid in self-monitoring and regulation of function. }\end{array}$ \\
\hline Adaptive & $\begin{array}{l}\text { Utilising strategies to minimise the symptoms and } \\
\text { impact of the cognitive deficit. }\end{array}$ & $\begin{array}{l}\text { The nature of the deficit is identified and compensatory strategies are } \\
\text { developed that allow the patient to adjust their approach in situations } \\
\text { where deficit is creating problems. }\end{array}$ \\
\hline Remedial & $\begin{array}{l}\text { Using interventions that focus on the cause of the } \\
\text { cognitive deficit. }\end{array}$ & $\begin{array}{l}\text { Use training routines that promote neuroplasticity to improve the } \\
\text { neurological pathways that are causing the deficit. }\end{array}$ \\
\hline Functional & $\begin{array}{l}\text { Structure the interventions to relate to the patients } \\
\text { particular lifestyle or work related needs. }\end{array}$ & $\begin{array}{l}\text { Adapt and apply the specific interventions to relevant activities that impact } \\
\text { upon the patient's lifestyle. }\end{array}$ \\
\hline
\end{tabular}




\subsubsection{Psychostimulants}

Methylphenidate is an amphetamine type agent that acts through catecholamine release to increase arousal, reaction time and attention. The minimal data in adults with primary brain tumours suggests a positive effect, but there are concerns over addictive potential. Meyers described an increase in cognitive functioning in a single arm study [18].

Modafinil is an alternative psychostimulant with potentially less addictive properties. It has been used in narcolepsy, and implemented in systemic malignancy as an agent to overcome persistent lethargy either from disease or treatment. A small randomized study of methylphenidate versus modafinil in 24 brain tumor patients and showed a beneficial effect in both arms with improved processing speed and executive function. There was a general benefit seen in both arms in terms of quality of life and mood. Doses of modafinil at $100 \mathrm{mg}$ twice daily were utilized in the study [19]. However a more recent Dutch randomised trial showed no additional benefit over placebo and there was a high withdrawal rate in the modafinil arm due to side-effects. The authors concluded that non-pharmacological interventions be assessed in future studies [20].

\subsubsection{Acetyl Cholinesterase Inhibitors}

Donepezil has been used in Alzheimer's dementia as a means to improve arousal. Early reports suggested a benefit in primary brain tumours; however a recent phase III study in over 190 patients with mild cognitive deficit after brain irradiation showed no significant improvement in global cognitive function. This multicentre study used donepezil at 5 - $10 \mathrm{mg}$ for 6 months and compared with placebo. There were some improved scores on selected assessments, but no overall benefit in a combined cognitive function score [21].

\subsubsection{Intercurrent Comorbidities}

Other related medical conditions may contribute to the cognitive deficit through a direct effect or indirectly via lethargy or mood disturbance. Conditions such as depression, complex partial seizure activity or endocrine insufficiency should be evaluated and managed appropriately [6]. Sedating anticonvulsant medications also may need to be reviewed and optimized depending on seizure control [11].

\subsection{Cognitive Rehabilitation Therapy}

Cognitive rehabilitation programmes have been an accepted part of therapy after traumatic brain injury and stroke management, but there is limited data in patients with primary brain tumours. With improved survival duration there is an increased need to optimize patient functioning after initial therapy, as well as to provide a means to protect or minimize subsequent delayed neurocognitive effects from treatment.

Cognitive Rehabilitation Therapy can be defined as "a systematic, functionally-oriented service of therapeutic cognitive activities, based on an assessment and understanding of the person's brain-behavior deficits. Services are directed to achieve functional changes by reinforcing, strengthening, or reestablishing previously learned patterns of behavior; or establishing new patterns of cognitive activity or compensatory mechanisms for impaired neurological systems" [15].

The effectiveness of cognitive rehabilitation in patients with primary brain tumours has been established by the Dutch randomized trial of a 6 week structured approach encompassing both compensatory and remedial interventions [22]. 140 symptomatic patients with favourable prognosis were randomised into either the individual programme or a waiting list for treatment (Table 2). Attention, executive function and memory were targeted in a programme encompassing education, compensatory strategies for daily activity and computer based process training. The weekly therapy sessions were combined with homework tasks including the computer exercises and logbook of experiences. Importantly the control

Table 2. Structure of cognitive rehabilitation therapy programme utilised in Dutch randomised trial [22].

\begin{tabular}{cl}
\hline \multirow{2}{*}{ Overall structure } & $\begin{array}{l}\text { Initial assessment session followed by } 6 \text { week programme involving weekly } 2 \text { hour therapy session and homework } \\
\text { activities Targeting attention, executive function and memory. }\end{array}$ \\
& Initial cognitive symptom screening followed by detailed neuropsychological assessment. \\
& Session 1: Education of cognitive problems and approach to rehabilitation. Identification of individual cognitive problems. \\
& Session 2: Understanding attention, executive function and memory; and general strategies to compensate. \\
& Session 3: Attention issues and factors that may impact on functioning. Strategies to improve attention. \\
& Session 4: Executive Function, specifically strategies to aid the planning of activities. \\
& Session 5: Memory and understanding where deficits are noted. Techniques to improve recall. \\
& Session 6: Summary of sessions and re-emphasis on specific issues. \\
Pdaptive training & $\begin{array}{c}\text { Process training using a computerized programme to train attention. This created scenarios training sustained, selective, } \\
\text { alternating and divided attention. Graded tasks implemented upon mastery of prior level and real-time feedback. } \\
\text { Remediation training } \\
\text { This was assigned both for identification of cognitive problems at home or work; and implementation of strategies } \\
\text { discussed in therapy sessions. Additionally the computerised process-training programme was undertaken at home. }\end{array}$ \\
\hline
\end{tabular}


groups were still provided with supportive care, which included telephone contact regarding their cognitive symptoms. At 3 months follow-up a statistically significant benefit was evident for subjective cognitive functioning and its perceived burden. At the 6-month followup, the intervention group also performed significantly better than the control group in regards to tests of attention and verbal memory. This brief structured cognitive rehabilitation intervention was thus able to improve patient experience and objective endpoints and can become the basis of an evidence-based approach to therapy.

An Italian randomized trial published in 2013 has confirmed the potential benefits of cognitive rehabilitation demonstrating improvements in visual attention and verbal memory in patients receiving an intensive programme early after surgery. 58 patients received 16 individual sessions over 4 weeks with both compensatory and computerised remedial training. At assessment after 4 weeks the control arm also gained an improvement in the time post-surgery; however the intervention arm had a greater recovery [23].

Although limited, the results of these two studies confirm that there is an improved cognitive function that can result from a structured neurocognitive rehabilitation programme. There is improvement that can be achieved, and the programme should incorporate a combination of both compensatory strategies and remediation, with an emphasis of relating these to functional activity. The initial programme can be of brief duration, and may involve home activities using computer-based activity. The benefit of further interventions after the initial programme to consolidate improvements is yet to be assessed, though it is noted that both randomized trials utilised follow-up programmes that were not assessed. The ability of these targeted interventions to generalise into global cognitive functioning is promising, given the measured objective and subjective improvements in most cognitive domains.

\subsection{Behavioral Therapies}

Cognitive behavioural therapies may have a role in deficits to minimize competing exacerbating effects such as stress, anxiety or lethargy. Relaxation, mindfulness and educational based programmes can be implemented to complement other strategies to optimize function [4,6]. A recent Australian study utilized an educational and behavioral approach in brain tumour patients with challenging behaviours. This consisted of skill-based training and environmental changes, and a workshop to train family members in compensatory strategy use. The study demonstrated a $71 \%$ reduction in target behaviour frequency following the programme [24].

\subsection{Technological Approaches}

Improvements in information technology and awareness of neuroplasticity have resulted in the development of multiple brain-training programmes aimed to increase working memory and attention [25]. Whilst most research has been undertaken using children or adults people without cognitive deficits, there is encouraging data in small series with traumatic brain injury, early neurodegenerative disorders and stroke that may be extrapolated to patients with primary brain tumours [26].

Uncertainties exist as to the ability of these individual managed exercises to provide durable or generalisable improvements in cognitive function; as well as programme compliance. These computer-based programmes however may be beneficial to augment remedial process training as demonstrated in the described Italian and Dutch studies.

\subsection{Exercise}

The relationship of exercise to improved neurocognitive status has been suggested in individual studies from traumatic brain injury and dementia-based research, but at present the data is limited [6,27]. Preclinical data also suggest that exercise may be associated with hippocampal neuronal regeneration via stem cell mobilization [28]. Whilst improvements have been noted with exercise in certain cognitive domains, this may possibly be related to the benefits in cardiorespiratory fitness. Systematic reviews however do not provide the evidence base for a direct positive association between exercise and cognitive function $[29,30]$.

\section{Conclusion}

Cognitive impairment is an important aspect to consider in regard to the assessment of the impact and morbidity of a primary brain tumour. Improved awareness of the potential cognitive effects in relation to neuroanatomical site may assist in the detection of subtle impairment that can affect recovery post diagnosis and treatment. A multidisciplinary approach to rehabilitation that encompasses adaptive, remedial, functional and metacognitive interventions can optimize outcome. A structured programme of cognitive rehabilitation involving multiple domains has been shown to increase aspects of attention executive function and working memory. Although evidence is limited, this programme may be augmented by selected pharmacological therapies, exercise and technological based training interventions.

\section{REFERENCES}

[1] R. Stupp, M. E. Hegi, W. P. Mason, et al., "Effects of Radiotherapy with Concomitant and Adjuvant Temozolomide versus Radiotherapy Alone on Survival in Glioblastoma in a Randomised Phase III Study: 5-Year Analysis of the EORTC-NCIC Trial," Lancet Oncology, Vol. 
10, No. 5, 2009, pp. 459-466.

http://dx.doi.org/10.1016/S1470-2045(09)70025-7

[2] G. Cairncross, M. Wang, E. Shaw, et al., "Phase III Trial of Chemoradiotherapy for Anaplasticoligodendroglioma: Long-Term Results of RTOG 9402,” Journal of Clinical Oncology, Vol. 31, No. 3, 2013, pp. 337-343.

http://dx.doi.org/10.1200/JCO.2012.43.2674

[3] M. van den Bent, A. Brandes, M. Taphoorn, et al., "Adjuvant Procarbazine, Lomustine, and Vincristine Chemotherapy in Newly Diagnosed Anaplastic Oligodendroglioma: Long-Term Follow-Up of EORTC Brain Tumor Group Study 26951,” Journal of Clinical Oncology, Vol. 31, No. 3, 2013, pp. 344-350.

http://dx.doi.org/10.1200/JCO.2012.43.2229

[4] K. Gehring, M. Sitskoorn, N. Aaronson and M. Taphoorn, "Interventions for Cognitive Deficits in Adults with Brain Tumours," Lancet Neurology, Vol. 7, No. 6, 2008, pp. 548-560.

http://dx.doi.org/10.1016/S1474-4422(08)70111-X

[5] D. M. Langenbahn, T. Ashman, J. Cantor, et al., “An Evidence-Based Review of Cognitive Rehabilitation in Medical Conditions Affecting Cognitive Function,” Archives of Physical Medicine and Rehabilitation, Vol. 94, No. 2, 2013, pp. 271-286.

http://dx.doi.org/10.1016/j.apmr.2012.09.011

[6] M. J. Taphoorn and M. Klein, "Cognitive Deficits in Adult Patients with Brain Tumours," Lancet Neurology, Vol. 3, No. 3, 2004, pp. 159-168. http://dx.doi.org/10.1016/S1474-4422(04)00680-5

[7] D. R. Johnson, A. M. Sawyer and C. A. Meyers, "Early Measures of Cognitive Function Predict Survival in Patients with Newly Diagnosed Glioblastoma,” Neuro-Oncology, Vol. 14, No. 6, 2012, pp. 808-816.

http://dx.doi.org/10.1093/neuonc/nos082

[8] O. Tucha, C. Smely, M. Preier and K. W. Lange, “Cognitive Deficits before Treatment among Patients with Brain Tumors,” Neurosurgery, Vol. 47, No. 2, 2000, pp. 324333.

http://dx.doi.org/10.1097/00006123-200008000-00011

[9] M. Klein, J. J. Heimans, N. K. Aaronson, et al., "Effect of Radiotherapy and Other Treatment-Related Factors on Mid-Term to Long-Term Cognitive Sequelae in LowGrade Gliomas: A Comparative Study,” Lancet, Vol. 360, No. 9343, 2002, pp. 1361-1368. http://dx.doi.org/10.1016/S0140-6736(02)11398-5

[10] K. Piil, M. Jarden, J. Jakobsen, et al., “A Longitudinal, Qualitative and Quantitative Exploration of Daily Life and Need for Rehabilitation among Patients with HighGrade Gliomas and Their Caregivers,” BMJ Open, Vol. 3, No. 7, 2013.

[11] M. Klein, N. Engleberts, H. van der Ploeg, et al., "Epilepsy in Low-Grade Gliomas: The Impact on Cognitive Function and Quality of Life,” Annals of Neurology, Vol. 54, No. 4, 2003, pp. 514-520.

http://dx.doi.org/10.1002/ana.10712

[12] M. Vargo, "Brain Tumor Rehabilitation,” American Journal of Physical Medicine \& Rehabilitation, Vol. 90, No. 5, 2011, pp. S50-S62.

http://dx.doi.org/10.1097/PHM.0b013e31820be31f
[13] G. Gillen, “Cognitive and Perceptual Rehabilitation: Optimizing Function,” Mosby Elsevier, St Louis Missouri, 2009.

[14] B. Zoltan, "Vision, Perception and Cognition. A Manual for the Evaluation and Treatment of the Adult with Acquired Brain Injury,” 4th Edition, Slack Incorporated, New Jersey, 2007.

[15] The Society for Cognitive Rehabilitation, "What Is Cognitive Rehabilitation?” 2013. www.societyforcognitiverehab.org

[16] M. Sohlberg and C. Mateer, "Cognitive Rehabilitation: An Integrative Neuropsychological Approach,” The Guildford Press, New York, 2001.

[17] S. Galbiati, M. Recta, V. Pastore, et al., "Attention Remediation Following Traumatic Brain Injury in Childhood and Adolescence," Neuropsychology, Vol. 23, No. 1, 2009, pp. 40-49. http://dx.doi.org/10.1037/a0013409

[18] C. Meyers, M. Weitzner, A. Valentine and V. Levin, "Methylphenidate Therapy Improves Cognition, Mood, and Function of Brain Tumor Patients," Journal of Clinical Oncology, Vol. 16, No. 7, 1998, pp. 2522-2527.

[19] K. Gehring, S. Y. Patwardhan and R. Collins, “A Randomized Trial on the Efficacy of Methylphenidate and Modafinil for Improving Cognitive Functioning and Symptoms in Patients with a Primary Brain Tumor," Journal of Neuro-Oncology, Vol. 107, No. 1, 2012, pp. 165-174. http://dx.doi.org/10.1007/s11060-011-0723-1

[20] F. Boele, L. Douw, M. de Groot, et al., "The Effect of Modafinil on Fatigue, Cognitive Functioning, and Mood in Primary Brain Tumor Patients: A Multicenter Randomized Controlled Trial," Neuro-Oncology, Vol. 15, No. 10, 2013, pp. 1420-1428. http://dx.doi.org/10.1093/neuonc/not102

[21] S. Rapp, D. Capp, A. Peiffer, et al., "Phase III Randomised Double-Blind, Placebo Controlled Trial of Donepezil in Irradiated Brain Tumour Survivors,” Journal of Clinical Oncology, Vol. 31, 2013.

[22] K. Gehring, M. Sitskoorn, C. Gundy, et al., "Cognitive Rehabilitation in Patients with Gliomas: A Randomized, Controlled Trial,” Journal of Clinical Oncology, Vol. 27, No. 22, 2009, pp. 3712-3722. http://dx.doi.org/10.1200/JCO.2008.20.5765

[23] C. Zucchella, A. Capone, V. Codella, et al., "Cognitive Rehabilitation for Early Post-Surgery Inpatients Affected by Primary Brain Tumor: A Randomized, Controlled Trial," Journal of Neuro-Oncology, Vol. 114, No. 1, 2013, pp. 93-100. http://dx.doi.org/10.1007/s11060-013-1153-z

[24] D. Whiting, G. Simpson, E. Koh, et al., "A Multi-Tiered Intervention to Address Behavioural and Cognitive Changes after Diagnosis of Primary Brain Tumour: A Feasibility Study,” Brain Injury, Vol. 26, No. 7-8, 2012, pp. 950961. http://dx.doi.org/10.3109/02699052.2012.661912

[25] A. Diamond and K. Lee, "Interventions Shown to Aid Executive Function Development in Children 4 to 12 Years Old," Science, Vol. 333, No. 6045, 2011, pp. 959963. http://dx.doi.org/10.1126/science.1204529

[26] A. Lundqvist, K. Gundstrom and J. Ronnberg, “Computerized Working Memory Training in a Group of Patients 
Suffering from Acquired Brain Injury,” Brain Injury, Vol. 24, No. 10, 2010, pp. 1173-1183.

http://dx.doi.org/10.3109/02699052.2010.498007

[27] A. Asher, "Cognitive Dysfunction among Cancer Survivors," Archives of Physical Medicine and Rehabilitation, Vol. 90, No. 5, 2011, pp. S16-S26.

[28] P. Eriksson, E. Perileva, T. Bjork-Eriksson, et al., "Neurogenesis in the Adult Hippocampus," Nature Medicine, Vol. 4, 1998, pp. 1313-1317. http://dx.doi.org/10.1038/3305

[29] N. Gates, M. Fiatarone Singh, P. Sachdev and M. Valen- zuela, "The Effect of Exercise Training on Cognitive Function in Older Adults with Mild Cognitive Impairment: A Meta-analysis of Randomized Controlled Trials," American Journal of Geriatric Psychiatry, Vol. 21, No. 11, 2013, pp. 1086-1097. http://dx.doi.org/10.1016/j.jagp.2013.02.018

[30] M. Angevaren, G. Aufdemkampe, H. Verhaar, et al., "Physical Activity and Enhanced Fitness to Improve Cognitive Function in Older People without Known Cognitive Impairment," Cochrane Database of Systematic Reviews, Vol. 2, 2008, Article ID: CD005381. 